\title{
UNSAFE REAR-END COLLISION AVOIDANCE IN ALZHEIMER'S DISEASE
}

\author{
Ergun Y. Uc ${ }^{1}$, Matthew Rizzo ${ }^{1,2,3}$, Steven W. Anderson ${ }^{1}$, \\ Qian Shi ${ }^{1,4}$, Jeffrey D. Dawson ${ }^{1,4}$ \\ University of Iowa \\ ${ }^{1}$ Division of Neuroergonomics, Department of Neurology \\ ${ }^{2}$ Department of Mechanical and Industrial Engineering \\ ${ }^{3}$ Public Policy Center \\ ${ }^{4}$ Department of Biostatistics \\ Iowa City, Iowa, USA \\ E-mail: ergun-uc@uiowa.edu
}

\section{PAPER NOT AVAILABLE}

\begin{abstract}
\section{OBJECTIVES}

Assess response of drivers with Alzheimer's disease (AD) to a traffic scenario creating the potential for a rear-end collision.
\end{abstract}

\section{BACKGROUND}

Rear-end crashes are among the most common crash types. Avoiding a crash requires continuous monitoring of neighboring vehicles, and anticipating and adjusting to changes in their speeds and positions, under pressure of time. This relies on visual perception, attention, memory, recognition of contextual cues such as approaching an intersection, and executive functions (decision making and implementation). AD impairs these processes, with clear implications for increased crash risk. (Rizzo et al., 2001)

\section{METHODS}

Sixty-one subjects with probable AD (defined by National Institute of Neurological and Communicative Disorders criteria) of mild severity, and 115 neurologically normal older adults were tested on a battery of visual, cognitive, and motor tests of abilities that are critical to safe automobile driving. Each participant also drove in a high-fidelity driving simulator. After a segment of uneventful driving, the participant suddenly encountered a lead vehicle stopped at a 4-way intersection waiting to turn left, posing a risk for a rear-end collision. The main dependent measure was the occurrence of an "improper response," which included crashing into the lead vehicle, swerving out of the traffic lane, or stopping abruptly and prematurely. The secondary dependent measure was "first reaction time."

\section{RESULTS}

Eighty-nine per cent of drivers with AD responded improperly to the stopped lead vehicle at the intersection compared to $65 \%$ of normal controls $(\mathrm{OR}=4.11,95 \%$ CI $1.71-9.88, \mathrm{P}=0.0007$, Fisher's exact test). Crash rates were similar in AD and normal controls (5\% and 3\%, 
respectively, $\mathrm{P}=0.4188$ ), however drivers with $\mathrm{AD}$ were at higher risk of stopping abruptly $(\mathrm{P}<0.0001)$ or prematurely $(\mathrm{P}=0.0115)$. These differences persisted after adjusting for differences in age, education, driving exposure, or level of simulator discomfort. Abrupt stopping increased the risk of being struck from behind by the following vehicle ( $\mathrm{P}=0.0262$, Fisher's exact test). The drivers with $\mathrm{AD}$ tended to respond slower to the encounter with the stopped lead vehicle at the intersection (first reaction time $11.5 \pm 3.5 \mathrm{sec}$ in $\mathrm{AD}$ and 10.6 $\pm 4.0 \mathrm{sec}$ in controls, $\mathrm{P}=0.0548$, Wilcoxon Rank Sum). Slower drivers were more likely to react improperly $(\mathrm{P}=0.0002)$. During a baseline segment, the drivers with $A D$ showed increased variability of their steering $(\mathrm{P}=0.0006)$ with a tendency for increased variability of their speed $(\mathrm{P}=0.0757)$. The drivers with $\mathrm{AD}$ performed worse in almost all cognitive, visual, and motor tests on the battery, consistent with their mild dementia. The risk of improper reactions increased significantly with worse performances on COGSTAT (a composite measure of cognition), Complex Figure Test Copy and Recall, Judgment of Line Orientation, Benton Visual Retention Test, Trail Making Subtest B, far visual acuity, contrast sensitivity, and Useful Field of View tests.

\section{CONCLUSIONS}

Drivers with AD reacted slower and were at a higher risk of responding unsafely in a simulated complex driving condition that posed a hazard for a rear-end collision compared to neurologically normal drivers. Although the drivers with AD were not statistically more likely to strike a lead vehicle, their slow and inappropriate reactions, such as abrupt stopping, appeared to increase the potential for being struck by another vehicle. These impairments can be explained by the cognitive and visual dysfunction in $\mathrm{AD}$, especially in the domains of visual perception, attention, memory, visuospatial abilities, and executive functions.

\section{ACKNOWLEDGMENTS}

This study was supported by: NIA AG 17717, NIA AG 15071.

\section{REFERENCES}

Rizzo, M., McGehee, D.V., Dawson, J.D., Anderson, S.N. (2001). Simulated car crashes at intersections in drivers with Alzheimer disease. Alzheimer Dis Assoc Disord., 15(1):10-20. 